Article

\title{
The Host Galaxies of Short GRBs as Probes of Their Progenitor Properties
}

\author{
Massimiliano De Pasquale \\ Department of Astronomy and Space Sciences, Faculty of Science, Istanbul University, 34119 Istanbul, Turkey; \\ m.depasquale@ucl.ac.uk
}

Received: 30 November 2018; Accepted: 22 January 2019; Published: 7 February 2019

\begin{abstract}
We present and discuss the properties of host galaxies of short Gamma-ray Burst (SGRBs). In particular, we examine those observations that contribute to the understanding of the progenitor systems of these explosions. Most SGRB hosts are found to be star forming objects, but an important fraction, $\sim 1 / 5$, of all hosts are elliptical with negligible star formation. Short bursts often occur at very large off-sets from their hosts, in regions where there is little or no underlying host light. Such results have enabled the community to test and improve the models for the production of short GRBs. In particular, the data are in favour of the merger of compact object binaries, provided that the kick velocities from the birth site are a few tens of $\mathrm{km} / \mathrm{s}$, and merger times of $\sim 1 \mathrm{Gyr}$.
\end{abstract}

Keywords: short GRBs; galaxies; compact object mergers

\section{Introduction}

It was discovered in 1993 [1], thanks to the Burst And Transient Source Experiment (BATSE) instrument onboard the Compton Gamma-ray Observatory, that Gamma-ray Bursts (GRBs) are divided into two classes of objects that show different observational properties. In particular, there is a clear bimodal distribution in the parameter $\mathrm{T}_{90}$, the time interval in which $90 \%$ of the counts of the prompt gamma-ray emission are collected, which shows two peaks at $\simeq 0.3$ and $\simeq 25 \mathrm{~s}$. Moreover, the spectra of the "short" GRBs are, on average, harder than the spectra of the "long" ones (LGRBs henceforth). While the exact values of peaks of the duration distributions and the divide depends on the observing instrument, and there is a certain degree of overlap in the distributions of $\mathrm{T}_{90}$ between the two classes, these early studies demonstrated that GRBs were not homogeneous. Differences emerged also when the first GRB afterglows were discovered and studied. Before the launch of the Neil Gehrels Swift Observatory mission [2], precise positions of SGRBs could not be obtained from their prompt emission (as was possible for LGRBs), which prevented the community from observing SGRB afterglows. However, in the rare cases in which a SGRB could be pinpointed with precision good enough to permit rapid follow up observations with narrow field instruments, afterglows were not seen in any electromagnetic band ([3-5] and references therein) All these pieces of information contributed to the idea that SGRB had different progenitors and "central engine" from those of long GRBs. In particular, the short duration indicated very compact progenitors and the lack of an afterglow pointed to a very thin circumburst medium (which would produce the observed afterglow emission). These characteristics pointed to the direction of mergers of binary neutron stars (NSs) or of NS-black hole (BH) systems [6-8]. To determine which physical models for short GRBs were viable, however, we still needed to find their afterglows. This way, we could constrain essential parts of the physics of these sources, such as the energy and the geometry of the ejecta. Furthermore, afterglows could point to the locations and the environments of the galactic systems that were the abodes of these explosions. The importance of positions and environs of cosmic sources cannot be overestimated: they indicate the nature of the progenitors. For example, LGRBs occur in star forming galaxies, in 
positions consistent with regions where new stars are born, while no LGRB has ever been associated with passive, early type galaxies (the possible exception of GRB 050219B [9], can be still explained as a chance superposition). This finding strongly supported the notion that the progenitor of LGRBs were massive stars, which did not live long and could not move much from their birth site. The situation changed with the discovery of SGRB afterglows, made possible thanks to Swift. Thanks to its very rapid repointing capabilities, Swift has been able to find the weak X-ray afterglows of short GRBs and thus determine the positions of these sources with an error of a few arcseconds; in many cases, Swift observations have also enabled the discovery of optical afterglows, which has led to positions of sub-arcsecond precision. Thanks to this accuracy, we have determined the host galaxies of tens of short GRBs, and have found the most likely hosts of many other events. In this review, we briefly summarize the results of systematic observations, and what they imply for the SRB progenitors.

\section{Statistics of SGRB Host Galaxies}

In the case of SGRBs with optical afterglows, the optical source enables us to pinpoint the GRB with a sub-arcsecond accuracy, and the bright galaxy in the immediate vicinity of the afterglow is generally regarded as the host of the event. However, as Reference [10] first found out, a few SGRB with optical afterglow occur in a relatively large region devoid of galaxies down to deep upper limits, typically of magnitude $m \gtrsim 25-26$. By means of probabilistic arguments, based on the size of such region, the density of field galaxies brighter than a certain magnitude, the afterglow position and the distance between a certain galaxy and the afterglow position, reference [11] derives the probability that a galaxy close by is not the host galaxy of the event (chance association, $\mathrm{P}_{c}$ ). Reference [11] regards a galaxy as the host of the event only if the $P_{c}$ is less than 0.05 . This allows the recovery a few more host GRB (see below); however, there are still SGRBs for which the methodology mentioned above yields a large probability (larger than $5 \%$, and sometimes as large as $~ 50 \%$ ) that surrounding galaxies are serendipitous objects, i.e., they are not the true host of the event. Furthermore, deep photometry with the Hubble Space Telescope and ground telescopes have not revealed nearby hosts for these events. A way to interpret these events is that one of the surrounding, visible galaxies is the host of the event, in the sense that the progenitor was born in it, but such a progenitor escaped the galaxy at high speed and reached a very large distance.

SGRB optical afterglows are, on average, one order of magnitude less luminous than the optical afterglows of LGRBs [12]. Out of the 68 SGRBs discovered by Swift up to May 2012, only $23(\simeq 1 / 3)$ have an optical afterglow. According to the prevailing model, the GRB afterglow emission is synchrotron radiation; the optical band is likely between the synchrotron peak frequency and cooling frequency. This is a regime in which the flux depends on both the density of the circumburst medium and the kinetic energy of the ejecta. If there is a relation between the density and the host galaxy type, optical afterglows might be biased against early type galaxies. Getting a picture as complete and unbiased as possible of the host galaxies of GRBs is critical for shedding light on the progenitors. In addition, the age of the stellar population tells us about the time it takes for the progenitor system to eventually produce a short GRB. For these reasons, reference [11] has also studied the SGRBs for which only an X-ray afterglow is known. The Swift X-ray telescope (XRT) can usually produce positions with an accuracy of $\simeq 1.5^{\prime \prime}$ at a $90 \%$ confidence level (CL). By examining the Swift archive of observations up to 2012, reference [11] finds a total of 36 objects (optical + X-ray identified) with $P_{c} \lesssim 0.05$. When breaking down this list on the basis of galaxy type (see their article and the references within), they find that $47 \%$ are late-type objects, $17 \%$ are early type galaxies, and $19 \%$ are "inconclusive", i.e., the data are not good enough to assess the kind of galaxy. Finally, 17\% SGRB are "host-less", i.e., they cannot be associated with a galaxy with $P_{c}<0.05$. When assigning the host-less GRBs to their most probable 
host galaxies, one recovers similar figures: $\simeq 50 \%, \simeq 20 \%$, and $\simeq 30 \%$ in the same categories as above (see Figure 1) ${ }^{1}$.

A Kolmogorov-Smirnov (KS) test does not show a correlation between the type of host galaxy of a short GRB and its duration [11]. This is of particular interest since, as mentioned in the Introduction, there is an overlap between the properties of SGRBs and LGRBs, and some events due to collapsars might have been erroneously included into the SGRB class. In principle, this could explain why one finds more star forming galaxies than elliptical objects among the hosts of SGRBs. However, this has been found not to be true. Reference [13] has shown that one can assign to a GRB event the probability that it is not to a collapsar, based on its duration. Reference [11] has attested that, even if we examine only SGRBs with very high probability $(>0.9)$ of not being caused by a collapsar, we find a ratio of star forming galaxies to passive galaxies even larger than that of the "complete" sample.

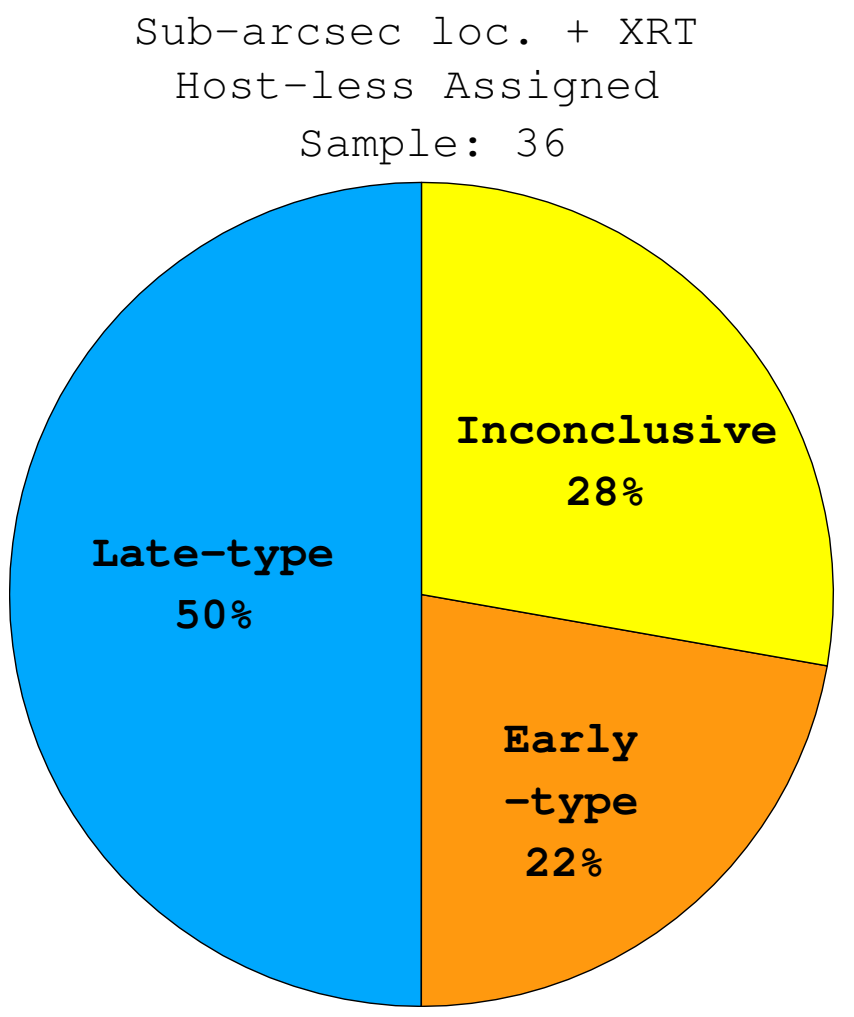

Figure 1. Pie diagram that shows the types of SGRB host galaxies. Source: Figure 13a in [11].

\section{The Location of SGRB Afterglows in Their Host Galaxies}

\subsection{Off-Set Distribution}

Reference [14] carried out a systematic study of the position of SGRBs with respect their host galaxies. Such an analysis was carried out by means of extensive Hubble Space Telescope imaging (see [14] for a detailed account of the procedure). It shows that the projected physical off-sets of the afterglows from the centres of the galaxies ranges from 0.5 to $75 \mathrm{kpc}$, while the median is $4.5 \mathrm{kpc}$. This is more than three times larger than the median off-sets for LGRBs (see Figure 2$)^{2}$, which is $\simeq 1.3 \mathrm{kpc}[15,16]$, but similar to the median off-sets of supernovae (SNe) Ia, which is $\simeq 3 \mathrm{kpc}$. However, SGRBs can be placed further away from their hosts than SN Ia; the latter are not found beyond $20 \mathrm{kpc}$ from their host, while 10\% of SGRBs are. Reference [14] has also studied the distribution of

\footnotetext{
http: / /iopscience.iop.org/article/10.1088/0004-637X/769/1/56/meta. http: / /iopscience.iop.org/article/10.1088/0004-637X/776/1/18/meta.
} 
the "host-normalized" off-sets, i.e., the ratios between the off-set of the afterglow and the host galaxy effective radii $r_{e}$. This is of great importance because the large physical off-sets of the afterglow per se may not indicate that SGRBs occur preferentially outside their host galaxies, as predicted in some models of compact binary mergers, if the host galaxies themselves are large. It is found that the host-normalized off-set distribution ranges from 0 to almost 15, while the median is 1.5, with only $25 \%$ of events at $\lesssim 1 \mathrm{r}_{e}$. This contrasts with the host-normalized $\mathrm{r}_{e} \simeq 1$ of LGRBs, core-collapse SNe, and even SN-Ia. The probability that LGRB and SGRB host-normalized off-sets are drawn from the same underlying population is only 3\%, while the same probability for SGRBs and SN-Ia is less than $10^{-3}$; these results indicate substantial differences between the progenitors of SGRBs and the other two classes of explosions.

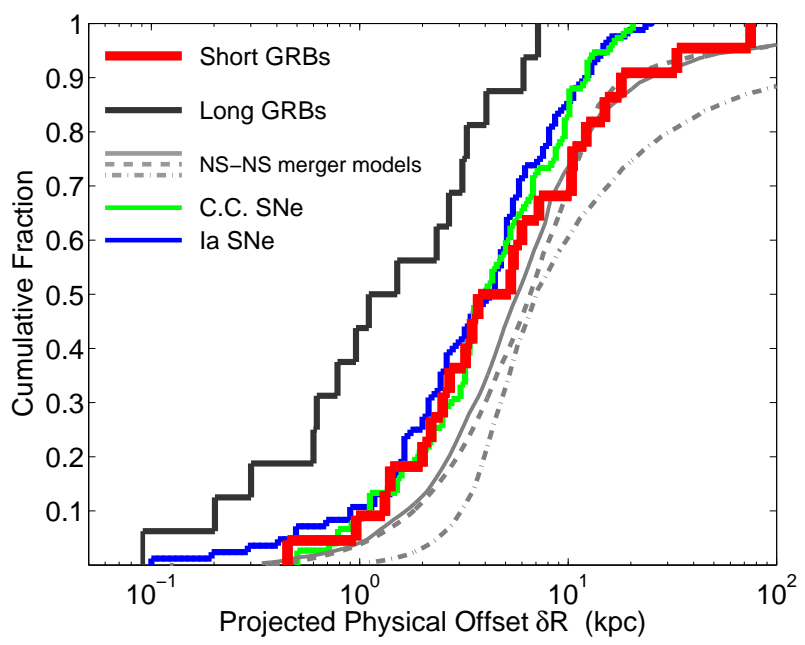

Figure 2. Cumulative distribution of projected physical off-sets for 22 short GRBs with sub-arcsecond positions (red; from [11]). Also shown are the cumulative distributions for long GRBs (black), core-collapse SNe (green), Type Ia SNe (blue); and predicted off-sets for NS-NS binaries (grey). Source: Figure 5 in [14].

\subsection{Light Fraction Distribution}

Reference [14] has also studied the so-called fractional flux for SGRB host, that is, the fraction of total host light in pixels fainter than or equal to the light in the pixel at the location of the transient [17]. Similar studies have already been performed in the case of hosts of core-collapse SNe, SN Ia, and LGRBs [16-18]. In their study of SGRB hosts, ref. [14] considered the fractional flux for UV light, with a rest-frame wavelength of less than $400 \mathrm{~nm}$, and that for optical light, with a rest-frame wavelength larger than $400 \mathrm{~nm}$. Naturally, this kind of study has to be restricted to those events that have an optical afterglow, giving a total of 20 SGRBs. Results are striking: $45 \%$ of SGRB are placed on the lowest level of optical brightness, i.e., 0 , and $55 \%$ are on the lowest level of UV emission. The median fractional optical flux is $\simeq 0.15$, while the median fractional UV flux is $\simeq 0$.

By comparing the SGRB fractional UV flux distribution with the host's galaxy own UV light distribution by means of a KS test, there is a quite low probability $p=0.01$ that the two distributions are drawn from the same intrinsic population, while this probability rises to 0.04 considering the optical light. These results show some similarities but differences as well with respect to those for SN Ia. About $34 \%$ of Ia SNe are placed on pixels that have zero UV flux; this percentage is lower but comparable to that for SGRBs. However, only $6 \%$ of SN Ia occur on regions of zero optical flux. The difference between SGRBs and LGRBs is even more obvious: the median fractional optical flux of LGRBs is 0.80 , while $5 \%$ of LGRBs occur on pixels with zero flux. A comparable analysis for core collapse supernovae yields results similar to those of LGRBs. 


\section{Implications of Galaxy Demographics, Off-Sets and Fractional Flux Distributions}

The ratio between star forming and passive hosts informs us about whether the SGRB rate is driven by star formation or stellar mass. A ratio 1:1 would indicate that the SGRB rate is driven by stellar mass alone, since the stellar mass in the two types of galaxies is roughly equal up to redshift $\simeq 1$; if instead the star formation rate influences the SGRB rate, one would expect a number of star forming, late galaxies larger than that of passive, early type galaxies. According to [11], the ratio of star forming galaxies to passive objects is $\simeq 2.5: 1$ (see Figure 1), quite different from 1:1, and an F-test run shows that there is only a $4 \%$ probability that the observed distribution of host galaxy type can be derived from a population in which the intrinsic ratio is 1:1. Only if all the objects in the "inconclusive" category were early type would become the ratio 1:1, but this circumstance is rather unlikely. As a consequence, one can infer that the SGRB rate is proportional to both the star formation rate and the stellar mass.

The number of hosts falling in the category of star forming galaxies is larger than those belonging to the class of early, elliptical galaxies, in which few to no stars are being formed. However, the latter still represent an important fraction of the total. This behaviour resembles that of type Ia supernovae, which take place in both star forming galaxies and elliptical galaxies. This similarity already suggests that the progenitors of SGRBs, like those of SN Ia, are evolved systems.

It is also possible to argue that, if the delay time, i.e., the time from the birth of the progenitor system until the SGRB, were several billion years, then one should prevalently find early type galaxies associated with SGRBs at $z \sim 0$. This does not appear to be the case, suggesting instead that the delay times are shorter. This conclusion is in agreement with a systematic study of the spectral energy distributions (SED) of SGRB hosts [11,19], which shows stellar populations with ages $\lesssim$ a few Gyr. As a consequence, the average delay time for SGRB should be $\sim 1$ Gyr.

The analysis of afterglow off-sets strongly indicates that the SGRB do not trace star forming regions and they are weakly, if at all, correlated with the stellar mass of the host galaxy. This indicates that they travel large distances from their birth sites. Moreover, their progenitors must be very different from those of LGRBs, and to some extent not similar to that those of SN Ia. A further insight into the nature of the progenitors can be derived if we compare the ages of the host galaxy populations and the off-sets of the SGRBs. Assuming that the delay between the birth of the progenitor system and the merger is comparable to the average stellar population age, one can infer the typical velocity at which the system moved from the birth site, that is, the "kick" velocity. First, reference [14] notes that there does not appear to be a correlation between the normalized off-set and the mass of the host galaxy (see the previous section). This result suggests that the gravity of the host galaxy does not play an important role in determining the kick velocity. Secondly, the distribution of off-sets leads to a distribution of projected speeds of the SGRB progenitors between 2 and $150 \mathrm{~km}^{-1} \mathrm{~s}^{-1}$. When considering the velocity dispersion of stars in the host, one finds projected kick velocities of $\simeq 20-140 \mathrm{~km}^{-1} \mathrm{~s}^{-1}$ with a median of $\simeq 60 \mathrm{~km}^{-1} \mathrm{~s}^{-1}$. This velocity range is consistent with the interval of kick velocities derived for NS-NS binaries in the Milky Way. Again with the aim of shedding light on the progenitors of short GRBs, it is interesting to compare the off-set observations with the predictions of population synthesis models of NS-NS binary mergers [20]. As is shown in Figure 2, the observed distribution is broadly consistent with that predicted one, although the simulations predict a somewhat larger off-set. This might be explained by the fact that, in order to build the off-set distribution, one needs an optical afterglow; this excludes $\simeq 2 / 3$ of SGRBs. In particular, an optical afterglow may be weaker and thus more likely to go undetected if the environment density is small (see above); this condition is more likely for GRBs occurring far away from the host galaxy. As a consequence, including events with X-ray only position may actually improve the agreement between the population synthesis and the real distribution of off-sets. 


\section{GW170817/GRB 170817A in the Context of SGRB Host Galaxies}

On 17 August 2017, the SGRB 170817A was detected by Fermi and INTEGRAL, only two seconds after the Gravitational Wave signal [21] which flagged the coalescence of a binary neutron star system was detected by aLIGO. Subsequent electromagnetic follow up observations showed a new optical transient where Fermi, INTEGRAL and the aLIGO error regions overlapped. These observations proved that the SGRB and the new electromagnetic source were indeed the outcome of the merger observed in the GW channel.

The host galaxy of the GRB 170817A/GW 170817 event was NGC 4993, at $\simeq 40$ Mpc from Earth. The property of this galaxy and the position of the source with respect to the galaxy body are consistent with those illustrated above. NGC 4993 is morphologically a lenticular galaxy, dominated by its bulge, although it also appears to be slightly distorted. The effective light radius of this object is $r_{e} \simeq 3 \mathrm{kpc}$ in the optical band, while it is $\simeq 2 \mathrm{kpc}$ for near-infrared observations [22]. The transient occurred at a projected distance of $\simeq 2 \mathrm{kpc}$ from the galaxy centre. These figures yield a normalized off-set of $\simeq 0.7$. The star formation rate of the galaxy is negligible, estimated to be less than a few thousandths of $\mathrm{M}_{\odot} \mathrm{yr}^{-1}$. According to [23], however, $\simeq 20$ percent of NGC 4993 stellar population is $\simeq 1$ Gyr old, while the rest is older than $5 \mathrm{Gyr}$. The fact that the merger occurred close to the centre of the host galaxy, together with the old age of stellar populations, suggests a relatively small kick velocity, although statistical and dynamical considerations indicate that it might have been as large as a few hundreds of $\mathrm{km} \mathrm{s}^{-1}$ [23]. At the position of GRB 170817A, no bright source is detected; the fractional flux is $\simeq 0.6$. This value is at the higher end of those seen in cosmological SGRBs (see previous sections). However, reference [23] points out that we may not detect the light from the faintest regions of the host galaxies of cosmological SGRBs, while the host galaxy of SGRB 170817A is located much closer to us.

\section{Conclusions}

About $50 \%$ or more of GRB host galaxies are late-type, star forming systems; only $\simeq 20 \%$ are definitely early-type, elliptical, passive objects. The remaining fraction is made of hosts we cannot identify or understand the features. These figures strongly indicate that the amount of star formation plays an important role in determining the SGRB rate, together with the stellar mass of the host. However, some SGRB hosts do not have star formation, and SGRBs often occur in the lowest UV luminosity pixel of their hosts. These facts indicate that SGRBs are not connected with recent star formation. Indeed, the age of stellar populations in the host galaxies is $\lesssim$ a few Gyr, suggesting that the timescale between the creation of the SGRB progenitor and the SGRB event itself is of the order of 1 Gyr. The median off-set of SGRBs is $4.5 \mathrm{kpc}$ (about three times that of LGRBs) and, together with the typical stellar population age, points out typical projected speeds of $\simeq 60 \mathrm{~km}^{-1} \mathrm{~s}^{-1}$ for the progenitors from their birth site to the final explosion. All the results described above indicate, or at least are consistent with, the scenario in which SGRB progenitors are binary systems made of two compact objects, either two neutron stars or a neutron star and a black hole.

Funding: This research was funded by the Research Fund of the University of Istanbul project number 30901.

Acknowledgments: M.D.P. thanks Alice Breeveld for her help with the manuscript, and Wen-Fai Fong for granting the right to re-use material in her articles. Figures 1 and 2 are (c) AAS, reproduced with permission.

Conflicts of Interest: The author declares no conflict of interest.

\section{References}

1. Kouveliotou, C.; Meegan, C.A.; Fishman, G.J.; Bhat, N.P.; Briggs, M.S.; Koshut, T.M.; Paciesas, W.S.; Pendleton, G.N. Identification of two classes of gamma-ray bursts. Astrophys. J. 1993, 413, L101-L104. [CrossRef]

2. Gehrels, N.; Chincarini, G.; Giommi, P.; Mason, K.O.; Nousek, J.A.; Wells, A.A.; White, N.E.; Barthelmy, S.D.; Burrows, D.N.; Cominsky, L.R.; et al. The Swift Gamma-Ray Burst Mission. Astrophys. J. 2004, 611, 1005-1020. [CrossRef] 
3. Klotz, A.; Boer, M.; Atteia, J.L. Observational constraints on the afterglow of GRB 020531. Astron. Astrophys. 2003, 404, 815-818. [CrossRef]

4. Hurley, K.; Berger, E.; Castro-Tirado, A.; Cerón, J.C.; Cline, T.; Feroci, M.; Frail, D.A.; Frontera, F.; Masetti, N.; Guidorzi, C.; et al. Afterglow Upper Limits for Four Short-Duration, Hard Spectrum Gamma-Ray Bursts. Astrophys. J. 2002, 567, 447-453. [CrossRef]

5. Gorosabel, J.; Fynbo, J.U.; Hjorth, J.; Wolf, C.; Andersen, M.I.; Pedersen, H.; Christensen, L.; Jensen, B.L.; Møller, P.; Afonso, J.; et al. Strategies for prompt searches for GRB afterglows: The discovery of the GRB 001011 optical/near-infrared counterpart using colour-colour selection. Astron. Astrophys. 2002, 384, 11-23. [CrossRef]

6. Narayan, R.; Paczyński, B.; Piran, T. Gamma-ray bursts as the death throes of massive binary stars. Astrophys. J. 1992, 395, L83-L86. [CrossRef]

7. Katz, J.L.; Canel, L.M. The Long and the Short of Gamma-Ray Bursts. Astrophys. J. 1996, 471, 915. [CrossRef]

8. Narayan, R.; Piran, T.; Kumar, P. Accretion Models of Gamma-Ray Bursts. Astrophys. J. 2001, 557, $949-957$. [CrossRef]

9. Rossi, A.; Piranomonte, S.; Savaglio, S.; Palazzi, E.; Michałowski, M.J.; Klose, S.; Hunt, L.K.; Amati, L.; Elliott, J.; Greiner, J.; et al. A quiescent galaxy at the position of the long GRB 050219A. Astron. Astrophys. 2014, 572, A47. [CrossRef]

10. Fong, W.; Berger, E.; Fox, D.B. Hubble Space Telescope Observations of Short Gamma-Ray Burst Host Galaxies: Morphologies, Offsets, and Local Environments. Astrophys. J. 2010, 708, 9. [CrossRef]

11. Fong, W.; Berger, E.; Chornock, R.; Margutti, R.; Levan, A.J.; Tanvir, N.R.; Tunnicliffe, R.L.; Czekala, I.; Fox, D.B.; Perley, D.A.; et al. Demographics of the Galaxies Hosting Short-duration Gamma-Ray Bursts. Astrophys. J. 2013, 769, 56. [CrossRef]

12. Kann, D.A.; Klose, S.; Zhang, B.; Covino, S.; Butler, N.R.; Malesani, D.; Nakar, E.; Wilson, A.C.; Antonelli, L.A.; Chincarini, G.; et al. The Afterglows of Swift-era Gamma-Ray Bursts. II. Type I GRB versus Type II GRB Optical Afterglows. Astrophys. J. 2011, 734, 96. [CrossRef]

13. Bromberg, O.; Nakar, E.; Piran, T. An Observational Imprint of the Collapsar Model of Long Gamma-Ray Bursts. Astrophys. J. 2012, 749, 110. [CrossRef]

14. Fong, W.; Berger, E. The Locations of Short Gamma-Ray Bursts as Evidence for Compact Object Binary Progenitors. Astrophys. J. 2013, 776, 18. [CrossRef]

15. Bloom, J.S.; Kulkarni, S.R.; Djorgovski, S.G. The Observed Offset Distribution of Gamma-Ray Bursts from Their Host Galaxies: A Robust Clue to the Nature of the Progenitors. Astron. J. 2002, 123, 1111. [CrossRef]

16. Lyman, J.D.; Levan, A.J.; Tanvir, N.R.; Fynbo, J.P.U.; McGuire, J.T.W.; Perley, D.A.; Angus, C.R.; Bloom, J.S.; Conselice, C.J.; Fruchter, A.S.; et al. The host galaxies and explosion sites of long-duration gamma ray bursts: Hubble Space Telescope near-infrared imaging. Mon. Not. R. Astron. Soc. 2017, 467, 1795-1817. [CrossRef]

17. Fruchter, A.S.; Levan, A.J.; Strolger, L.; Vreeswijk, P.M.; Thorsett, S.E.; Bersier, D.; Burud, I.; Cerón, J.C.; Castro-Tirado, A.J.; Conselice, C.; et al. Long gamma-ray bursts and core-collapse supernovae have different environments. Nature 2006, 441, 463-468. [CrossRef] [PubMed]

18. Svensson, K.M.; Levan, A.J.; Tanvir, N.R.; Fruchter, A.S.; Strolger, L.G. The host galaxies of core-collapse supernovae and gamma-ray bursts. Mon. Not. R. Astron. Soc. 2010, 405, 57-76. [CrossRef]

19. Leibler, C.N.; Berger, E. The Stellar Ages and Masses of Short Gamma-ray Burst Host Galaxies: Investigating the Progenitor Delay Time Distribution and the Role of Mass and Star Formation in the Short Gamma-ray Burst Rate. Astrophys. J. 2010, 725, 1202. [CrossRef]

20. Belczynski, K.; Perna, R.; Bulik, T.; Kalogera, V.; Ivanova, N.; Lamb, D.Q. A Study of Compact Object Mergers as Short Gamma-Ray Burst Progenitors. Astrophys. J. 2006, 648, 1110. [CrossRef]

21. Abbott, B.P.; Abbott, R.; Adhikari, R.X.; Ananyeva, A.; Anderson, S.B.; Appert, S.; Arai, K.; Araya, M.C.; Barayoga, J.C.; Barish, B.C.; et al. Multi-messenger Observations of a Binary Neutron Star Merger. Astrophys. J. 2017, 848, 12. [CrossRef] 
22. Im, M.; Yoon, Y.; Lee, S.K.J.; Lee, H.M.; Kim, J.; Lee, C.U.; Kim, S.L.; Troja, E.; Choi, C.; Lim, G.; et al. Distance and Properties of NGC 4993 as the Host Galaxy of the Gravitational-wave Source GW170817. Astrophys. J. 2017, 849, 16. [CrossRef]

23. Levan, A.J.; Lyman, J.D.; Tanvir, N.R.; Hjorth, J.; Mandel, I.; Stanway, E.R.; Steeghs, D.; Fruchter, A.S.; Troja, E.; Schrøder, S.L.; et al. The Environment of the Binary Neutron Star Merger GW170817. Astrophys. J. 2017, 848, 28. [CrossRef]

(C) 2019 by the author. Licensee MDPI, Basel, Switzerland. This article is an open access article distributed under the terms and conditions of the Creative Commons Attribution (CC BY) license (http://creativecommons.org/licenses/by/4.0/). 